\title{
SUPPLEMENTARY MATERIAL \\ EFFECTS OF FLAVONOID DERIVATIVES ON HUMAN MICROVASCULAR ENDOTHELIAL CELLS
}

\author{
Eleonora Bassino1, Susanna Antoniotti1, Franco Gasparri2 ${ }^{2}$ \& Luca Munaron ${ }^{1}$ \\ ${ }^{1}$ Department of Life Sciences \& Systems Biology, University of Torino, ${ }^{2}$ Department of Pharmacy (DIFARMA), \\ University of Salerno, Italy.
}

\begin{abstract}
Some natural compounds, including flavonoids, are active in vasculature re-growth during hair follicle disruption, but their effects have not been yet evaluated directly on microvascular endothelial cells.

Skin vascularization regulates the physiological blood supply required for hair growth and its dysregulation is the basis of several human diseases. Follicle-derived VEGF release from follicular keratinocytes promotes perifollicular vascularization and increases follicle and hair size, while blockade of VEGF-mediated angiogenesis leads to impaired hair growth.

Here we tested three flavonoids, Visnadin (VSD), Hesperidin (HSP) and Baicalin (BC), on cultured human microvascular endothelial cells (HMEC), comparing their effects with Minoxidil (MXD), a synthetic drug broadly used in the treatment of Androgenetic Alopecia. The response to these compounds was assayed in terms of endothelial survival, proliferation, tubulogenesis and proangiogenic signalling.

We show that BC promotes HMEC proliferation, while both VSD and MXD enhance tubulogenesis. Interestingly, only HSP increases VEGFR-2 phosphorylation.
\end{abstract}

Keywords: natural products, flavonoids, endothelial cells, angiogenesis, hair follicle 


\section{Experimental}

Cell Culture

Human microvascular endothelial cell line (HMEC) was purchased from Lonza, grown in EGM 2MV medium (Lonza) and maintained at $37^{\circ} \mathrm{C}$ in a humidified atmosphere of $5 \% \mathrm{CO}_{2}$.

\section{Drugs}

All drugs (MXD, VSD, BC and HSP) have been provided by Rottapharm-Madaus.

\section{Cell viability and proliferation}

For survival and proliferation assays HMEC were thawed from frozen stock and seeded in 96 well plates at a density of $5000 / \mathrm{cm}^{2}$ (survival and proliferation). Cell viability was analyzed after $24 \mathrm{~h}$ of incubation with MXD or the different flavonoids-derived compounds. For proliferation assays cell medium was discarded after $18 \mathrm{~h}$, then complete medium was replaced with Dulbecco's Modified Eagle Medium (DMEM) 2\% Fetal Cow Serum (FCS) to reduce cell proliferation for $24 \mathrm{~h}$ and replaced with DMEM 2\% FCS alone (basal conditions) or with different compounds. Cell number was evaluated after $48 \mathrm{~h}$ of treatment.

Cell viability and proliferation were evaluated by the CellTiter 96® AQueous Non-Radioactive Cell Proliferation Assay (Promega, Madison, WI, USA), using 3-(4,5-dimethylthiazol-2-yl)-5-(3carboxymethoxyphenyl)-2-(4-sulfophenyl)-2Htetrazolium, inner salt (MTS). In brief, MTS is a homogeneous colorimetric method for determining the number of viable cells in proliferation, cytotoxicity or chemo-sensitivity assays. MTS is bio-reduced by cells into a formazan product that is soluble in a tissue culture medium. The absorbance of the formazan product at $490 \mathrm{~nm}$ can be measured directly from 96-well assay plates without additional processing. The quantity of the formazan product was measured in a Bio-Rad microplate reader Model 680 (Bio-Rad, Hercules, CA, USA) at $490 \mathrm{~nm}$, as absorbance is directly proportional to the number of viable cells in culture.

\section{Tubulogenesis}

Matrigel (Collaborative Biomedical Products, USA) was used to make up a basement membrane matrix gel solution for cell suspension. Subconfluent HMEC were trypsinized and $5.0 \times 10^{4}$ cells were added to each well of a chilled 24 -well plate and allowed to gel for $30 \mathrm{~min}$ at $37^{\circ} \mathrm{C}$ in a humidified $5 \% \mathrm{CO}_{2}$ atmosphere. HMEC, at the different experimental conditions, were maintained in a stable humidified $5 \% \mathrm{CO}_{2}$ atmosphere for all the experimental time course $(18 \mathrm{~h})$. Images acquisition and statistical analysis were performed with Metafluor and ImageJ softwares, 
respectively. Tubulogenic index (expressed in A.U.) has been obtained as vessel length $x$ number of junctions.

\section{Immunoblotting}

For immunoblotting experiments HMEC were treated with the different natural compounds for 24 h. In particular, HMEC were grown in EGM 2-MV medium containing 10\% FCS until $80 \%$ confluent. Cells were detached and suspended in ice-cold PBS containing the following protease inhibitors: $2 \mu \mathrm{g} / \mathrm{mL}$ aprotinin, $1 \mathrm{mmol} / \mathrm{L} \mathrm{Na}$ orthovanadate, $0.1 \mathrm{mmol} / \mathrm{L}$ phenylmethylsulfonyl fluoride, and $10 \mathrm{mmol} / \mathrm{L} \mathrm{NaF}$. Cells were centrifuged for 15 minutes at $1,000 \times g$ and resuspended in lysis buffer (Sigma) in the presence of protease inhibitors and frozen at $-80^{\circ} \mathrm{C}$ for at least 2 hours before use. Protein concentration was determined using the BCA protein assay (Invitrogen) following the manufacturer's instructions. Conditions for SDS-PAGE and Western blotting were as described previously. Polyvinylidene fluoride membranes were blocked and incubated for overnight with rabbit anti-P-Flk1 antibody (1:100) (Santa Cruz, Biotechnology) and the mouse IgG2 $\alpha$ anti- $\beta$ actin (Sigma, St Louis, MO, USA) antibodies. The membrane was then washed with TBS containing $0.1 \%$ Tween 20 and incubated as required with the HRP-conjugated anti-mouse or antirabbit IgG antibodies (Amersham, Piscataway, NJ, USA). Electrochemiluminescence was conducted using the SuperSignal West Pico chemiluminescent substrate (Pierce, Rockford, IL, USA).

\section{Statistics}

Statistical significance of all experiments was evaluated by Kaleidagraph software (Synergy Software, USA) using nonparametric Wilcoxon test. The Wilcoxon test was chosen for the proliferation and survival assay analysis because for each condition in each experiment five biological replicates were done and they were not normally distributed. The Wilcoxon test was also used for cell tubulogenesis analysis because the percentages of tubulogenesis were not normally distributed. All values are presented as the mean \pm standard error (SE). Results with $p$-values $<0.05$ were considered statistically significant and shown with *. 
a)

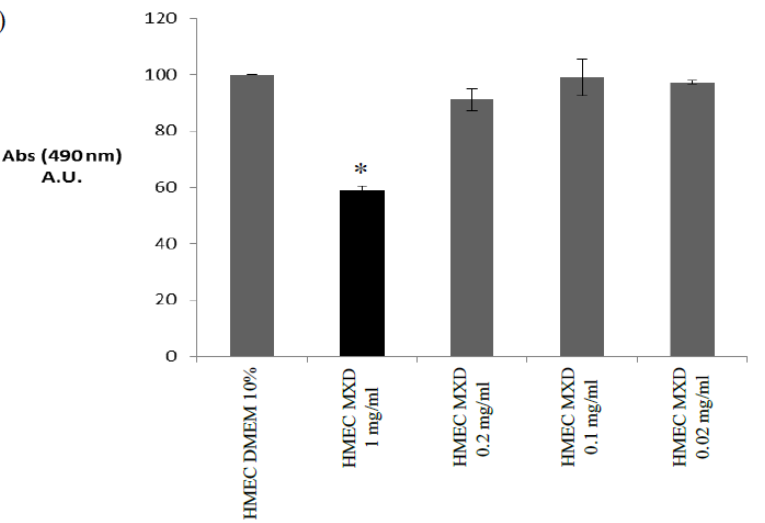

b)

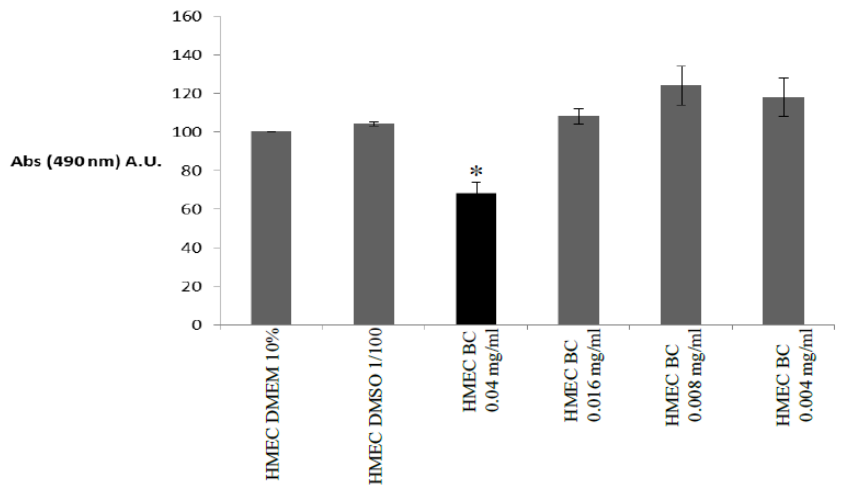

c)

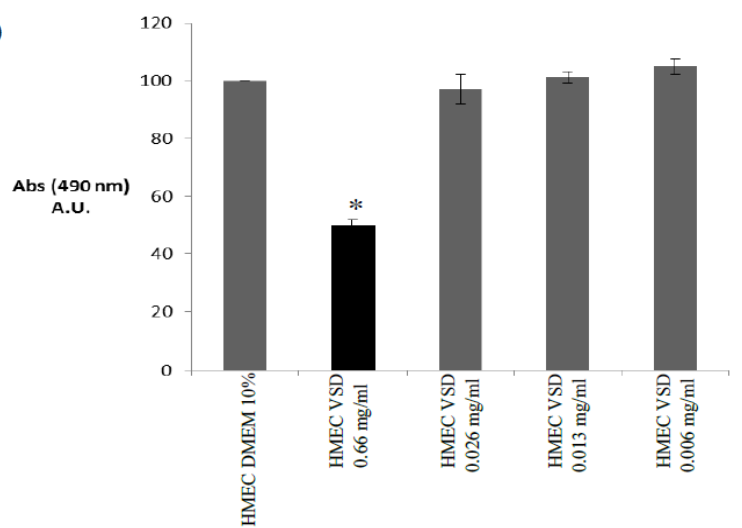

d)

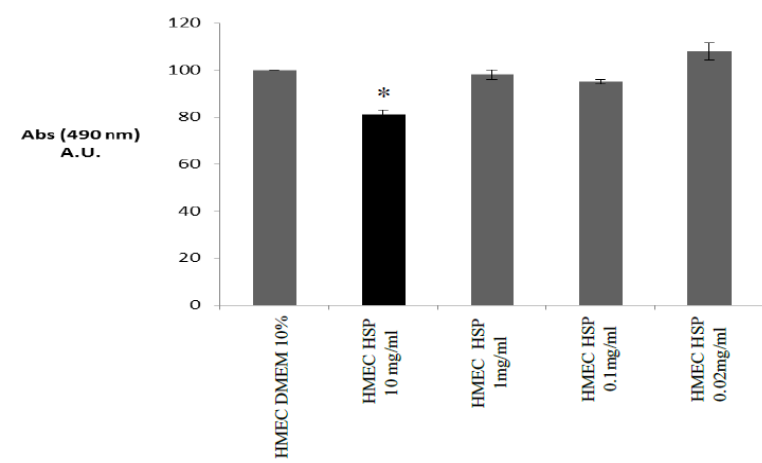

f)

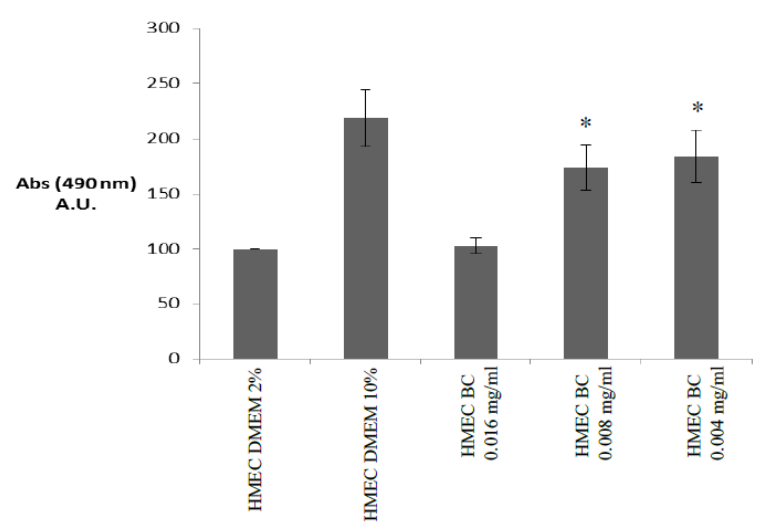

h)

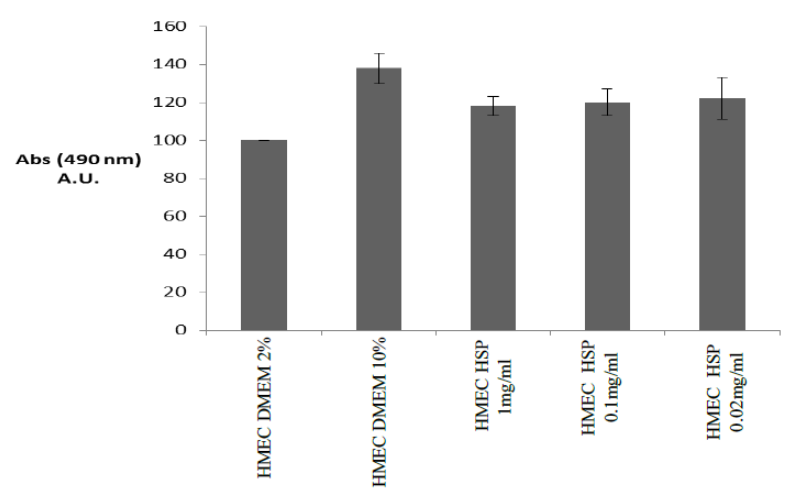


Figure S1.

$a-d$. Cell viability measured by MTS test. All values are presented as the mean \pm standard error (SE).

Results with $p$-values $<0.05$ were considered statistically significant: $* \mathrm{p}<0.05$ (Wilcoxon test). $e-h$. Cell proliferation measured by MTS test. All values are presented as the mean \pm standard error (SE). Results with $p$-values $<0.05$ were considered statistically significant: ${ }^{*} p<0.05$ (Wilcoxon test). 
a)

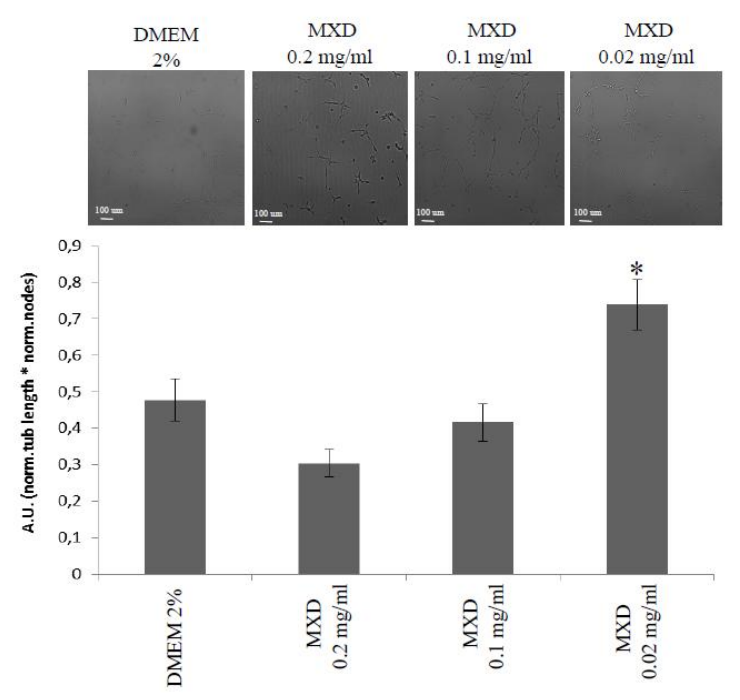

c)

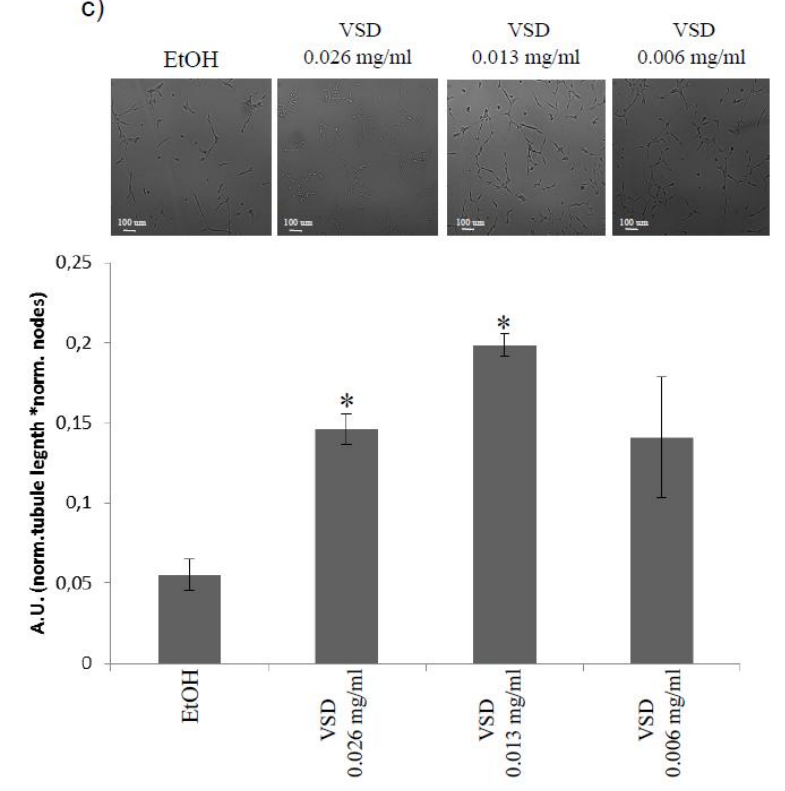

e)

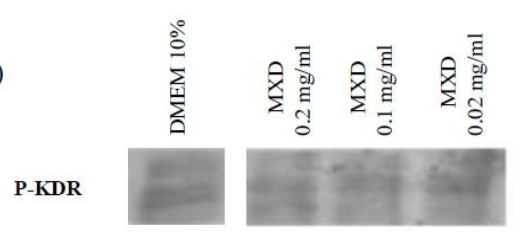

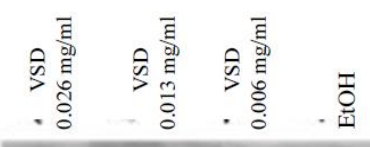

P-KDR

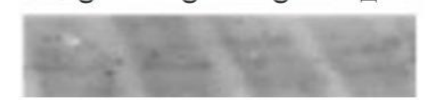

b)

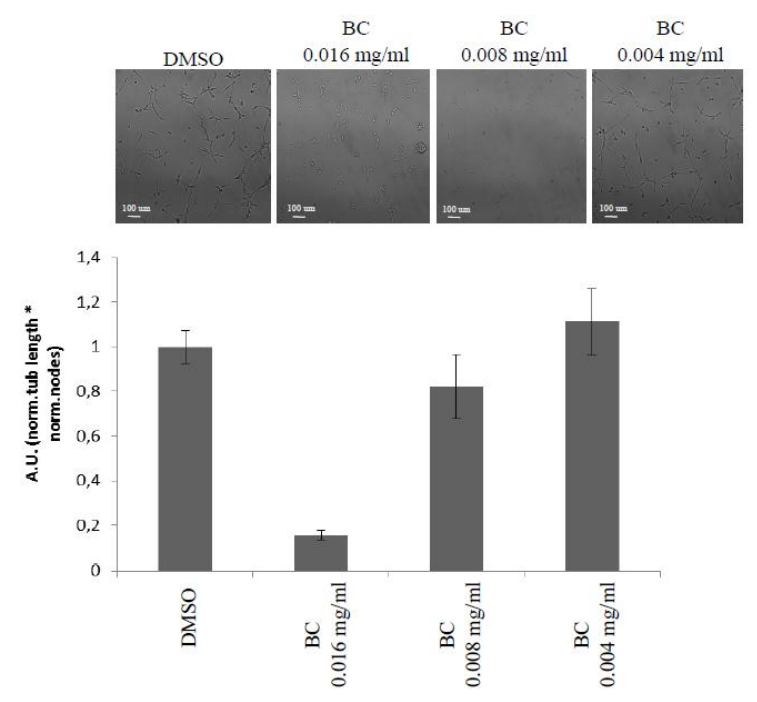

d)
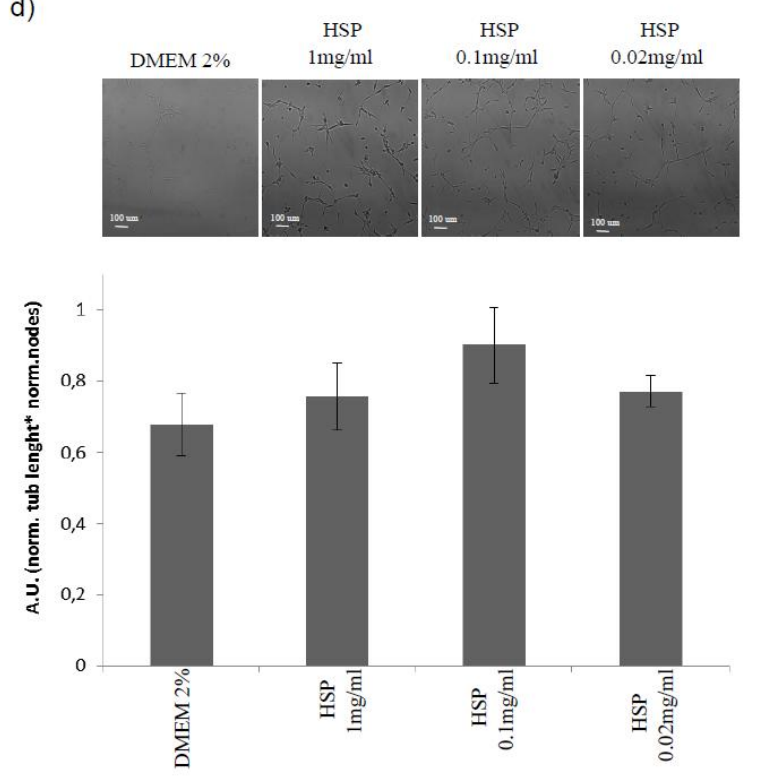

P-KDR
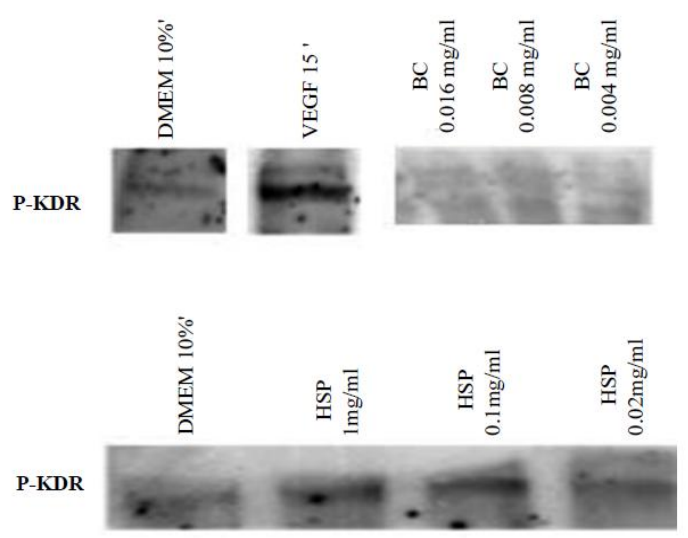

Figure S2. 
$a-d$. In vitro tubulogenesis of HMECs cultured in 3D matrigel. Tubulogenic indexes (see Methods) are presented as the mean \pm standard error (SE). Representative images were used to quantify the tubulogenic effect on HMEC.

Tubule length was manually measured and tubulogenic index (expressed in A.U.) has been obtained as tubule length $x$ number of junctions. Results with $p$-values $<0.05$ were considered statistically significant: $* \mathrm{p}<0.05$ (Wilcoxon test).

$e$. Tyrosine phosphorylation of VEGFR-2 (KDR) evaluated by western blotting. Cells were treated with VEGF or chemical compounds at different concentrations for $2 \mathrm{~h}$. 